\title{
Impact of Maintenance Therapy Following Induction Immunochemotherapy for Untreated Advanced Non-Small Cell Lung Cancer Patients
}

Ryota Nakamura ( $\sim$ ryotan@koto.kpu-m.ac.jp )

Kyoto Prefectural University of Medicine: Kyoto Furitsu Ika Daigaku https://orcid.org/0000-0001-95192276

\section{Tadaaki Yamada}

Kyoto Prefectural University of Medicine: Kyoto Furitsu Ika Daigaku

Kenji Morimoto

Kyoto Prefectural University of Medicine: Kyoto Furitsu Ika Daigaku

\section{Akira Nakao}

Fukuoka University: Fukuoka Daigaku

\section{Yasuhiro Goto}

Fujita Health University: Fujita Ika Daigaku

\section{Yuri Ogura}

Kyoto Daini Sekijuji Byoin

\section{Takayuki Takeda}

Kyoto Daini Sekijuji Byoin

Chieko Takumi

Kyoto Daiichi Sekijuji Byoin

\section{Keisuke Onoi}

Uji-Tokushukai Medical Center

\section{Yusuke Chihara}

Uji-Tokushukai Medical Center

\section{Ryusuke Taniguchi}

Matsushita Memorial Hospital

\section{Takahiro Yamada}

Matsushita Memorial Hospital

\section{Osamu Hiranuma}

Otsu City Hospital

\section{Satomi Tanaka}

Kyoto Prefectural University of Medicine: Kyoto Furitsu Ika Daigaku Yoshie Morimoto

Kyoto Prefectural University of Medicine: Kyoto Furitsu Ika Daigaku 


\section{Masahiro Iwasaku}

Kyoto Prefectural University of Medicine: Kyoto Furitsu Ika Daigaku

\section{Shinsaku Tokuda}

Kyoto Prefectural University of Medicine: Kyoto Furitsu Ika Daigaku

\section{Yoshiko Kaneko}

Kyoto Prefectural University of Medicine: Kyoto Furitsu Ika Daigaku

\section{Junji Uchino}

Kyoto Prefectural University of Medicine: Kyoto Furitsu Ika Daigaku

\section{Koichi Takayama}

Kyoto Prefectural University of Medicine: Kyoto Furitsu Ika Daigaku

\section{Research Article}

Keywords: non-small-cell lung cancer, immunochemotherapy, maintenance therapy, pemetrexed, immune checkpoint inhibitor

Posted Date: September 27th, 2021

DOl: https://doi.org/10.21203/rs.3.rs-866475/v1

License: (c) (i) This work is licensed under a Creative Commons Attribution 4.0 International License. Read Full License

Version of Record: A version of this preprint was published at Journal of Cancer Research and Clinical Oncology on November 26th, 2021. See the published version at https://doi.org/10.1007/s00432-02103866-2. 


\section{Abstract \\ Purpose}

The primary objective of this study was to identify the potential predictors to assess impact of maintenance therapy after induction immunochemotherapy in the real-world setting of patients with advanced non-small cell lung cancer (NSCLC).

\section{Methods}

We retrospectively identified 152 patients with advanced NSCLC who received immunochemotherapy at eight hospitals in Japan between January 2019 to December 2019. Patients who received at least four cycles of induction immunochemotherapy and one cycle of maintenance therapy with immunecheckpoint inhibitors were included. We investigated the biomarkers for progression-free survival (PFS) for maintenance therapy after induction immunochemotherapy.

\section{Results}

Out of the 92 patients with advanced NSCLC included in the study, 42 received maintenance therapy with cytotoxic agents whereas 50 received maintenance therapy without cytotoxic agents. Among those who received maintenance therapy without cytotoxic agents, responders to prior immunochemotherapy had significantly longer PFS than non-responders $(p=0.004)$, except those with maintenance therapy with cytotoxic agents. In non-responders to prior immunochemotherapy, patients with maintenance therapy with cytotoxic agents had significantly longer PFS than those with maintenance therapy without cytotoxic agents (log-rank $p=0.007$ ), whereas among responders to prior immunochemotherapy, there was no significant difference in PFS for different maintenance regimens (log-rank $p=0.31$ ).

\section{Conclusions}

This retrospective study showed that response to prior immunochemotherapy was associated with clinical outcomes among patients with advanced NSCLC who received maintenance therapy.

\section{Introduction}

Lung cancer is the leading cause of cancer-related deaths worldwide (1). Novel combination therapies, such as immune checkpoint inhibitors (ICls) plus chemotherapy or dual-immunotherapy with or without chemotherapy have recently been introduced as standard treatments for patients with advanced nonsmall cell lung cancer (NSCLC) (2-6). Furthermore, follow up maintenance therapy has been standard treatment for patients with advanced NSCLC who responded positively to previous immunochemotherapy $(7-10)$. 
In the KEYNOTE-189 trial, the addition of pembrolizumab to platinum-based chemotherapy followed by pemetrexed plus pembrolizumab maintenance had demonstrated higher overall survival (OS) and progression-free survival (PFS), compared to platinum-based chemotherapy followed by pemetrexed among patients with previously untreated metastatic nonsquamous NSCLC (8). Contrastingly, the phase III PARAMOUNT trial provided evidence for better results with pemetrexed continuation maintenance therapy as compared with placebo (11). This evidence suggests that pemetrexed maintenance therapy could be a useful therapeutic strategy for patients with advanced NSCLC who responded positively to previous immunochemotherapy, irrespective of the induction immunochemotherapy.

However, a previous prospective cohort study demonstrated that pemetrexed maintenance therapy induced acute kidney injury in approximately $30 \%$ of patients with advanced NSCLC and promoted treatment discontinuation among $20 \%$ of them (12). Another retrospective cohort study disclosed that patients with NSCLC on pemetrexed-based long term-treatment were at risk of developing renal impairment, despite renal function remaining stable following pemetrexed treatment induction. This suggested that a long duration of pemetrexed-based chemotherapy exposure might be harmful (13). Therefore, the decision to continue cytotoxic agents with maintenance following induction immunochemotherapy in patients with advanced NSCLC remains unclear.

Thus, the primary objective of this study was to identify the potential predictors to assess the impact of chemotherapy containing maintenance therapy after induction immunochemotherapy in the real-world setting of patients with advanced NSCLC.

We present the following article in accordance with the STROBE reporting checklist.

\section{Materials And Methods}

\subsection{Patients}

In the present study, 152 patients with advanced NSCLC on a combination therapy of immunotherapy and chemotherapy at eight institutions in Japan (University Hospital Kyoto Prefectural University of Medicine, Japanese Red Cross Kyoto Daiichi Hospital, Japanese Red Cross Kyoto Daini Hospital, UjiTokushukai Medical Center, Otsu City Hospital, Matsushita Memorial Hospital, Fukuoka University Hospital, and Fujita Health University Hospital) between January and December 2019 were retrospectively identified. Patients who received at least four cycles of induction therapy and one cycle of maintenance therapy were included. The medical records of each patient were reviewed to collect the following data: age at the start of induction therapy; sex; smoking status; treatment regimens; body mass index (BMI) at the start of induction therapy; laboratory findings at the start of maintenance therapy; Eastern Cooperative Oncology Group Performance Status (ECOG-PS) at the start of induction therapy; disease staging classified using the TNM stage classification system version 8; histological subtypes; epidermal growth factor receptor (EGFR) mutation status; anaplastic lymphoma kinase (ALK) fusion status; programmed death-ligand 1 (PD-L1) expression level in tumors measured using the PD-L1 IHC 
22C3 pharmDx assay (Agilent Technologies, Santa Clara, CA, USA); best overall response (BOR) without confirmation evaluated by each attending physician according to the Response Evaluation Criteria in Solid Tumors (RECIST) version 1.1; and PFS of maintenance therapy.

\subsection{Ethical Consideration}

The study was conducted in accordance with the Declaration of Helsinki (as revised in 2013). The study protocol was approved by the Ethics Committees of the Kyoto Prefectural University of Medicine (approval no. ERB-C-1803) and each of the participating hospitals. Informed consent was not required due to the retrospective nature of the study, and used the official website as an opt-out method, which was also approved by the ethics committee of each hospital.

\subsection{Statistical analysis}

We retrospectively collected data on September 30, 2020. Patients who were alive and progression free were censored at the date of last follow-up update. Student's t-test was used to compare the means of continuous variables (such as age). To compare the proportions of categorical variables (such as sex) between the groups, Fisher's exact test was employed. Survival curves were calculated with the KaplanMeier method, and differences were compared by using the log-rank test. PFS of maintenance therapy was calculated from the date of initiation of maintenance therapy to the date of disease progression or death from any cause. Cox proportional hazards models were used to calculate the hazard ratios (HRs) and $95 \%$ confidence intervals $(\mathrm{Cls})$. The variables with $p<0.10$ in univariate analysis were included in the multivariate analysis. Patients whose laboratory data was missing or whose BOR was not evaluable (NE) were excluded from the analysis. The cut-off point of PFS for patients without chemotherapy for maintenance therapy was set at 90 days, based on PFS of the control arm in the PARAMOUNT trial (14). Responders were defined as patients who achieved complete response (CR) and partial response (PR), and non-responders were defined as patients who had stable disease (SD). Maintenance therapy with cytotoxic agents (cytotoxic maintenance) was defined as maintenance therapy with pemetrexed and ICls, and maintenance therapy without cytotoxic agents (non-cytotoxic maintenance) was defined as maintenance therapy with ICls alone or in combination with anti-VEGF antibody bevacizumab. Neutrophilto-lymphocyte ratio (NLR) was defined as the absolute neutrophil count divided by the absolute lymphocyte count. The cut-off value of NLR was set at five, based on a previous study (15). Statistical analyses were performed using EZR statistical software (version 1.42. Division of Hematology, Saitama Medical Center, Jichi Medical University, Japan) (16). All statistical tests were two-tailed and $p$ value < 0.05 was considered significant.

\section{Results}

\subsection{Patient characteristics}

A total of 152 patients with advanced NSCLC on a combination of immunotherapy plus chemotherapy at eight hospitals in Japan from January to December 2019 were identified. Ninety-three of the identified 
patients who had received at least four cycles of induction therapy and one cycle of maintenance therapy were included in the present study. Fifty patients received non-cytotoxic maintenance and 43 patients received cytotoxic maintenance. One patient receiving cytotoxic maintenance was excluded from analysis due to missing laboratory data (Fig. 1). The median follow-up time after initiation of maintenance therapy was 9.8 months.

Patient characteristics classified by maintenance regimens are shown in Table 1. Median age of the patients with cytotoxic maintenance and those with non-cytotoxic maintenance was 69 years (range 4579 ) and 68 years (range 39-79), respectively. About 95.2\% $(n=40)$ of the patients received cytotoxic maintenance of platinum with pemetrexed plus pembrolizumab while the remaining received carboplatin with pemetrexed plus atezolizumab. The regimens in the patients with non-cytotoxic maintenance were carboplatin with paclitaxel, atezolizumab, and bevacizumab $(26.0 \%, n=13)$, carboplatin with paclitaxel or nab-paclitaxel plus pembrolizumab $(72.0 \%, n=36)$, and carboplatin with nab-paclitaxel plus atezolizumab $(2.0 \%, n=1)$. All patients receiving cytotoxic maintenance suffered from adenocarcinoma. The patients with non-cytotoxic maintenance showed a higher proportion of squamous cell carcinoma $(52.0 \%, \mathrm{n}=26)$.

\subsection{Association of clinical outcomes of maintenance therapy to induction immunochemotherapy}

The results of univariate and multivariate analyses for PFS of maintenance therapy using Cox proportional hazards models are presented in Table 2. Multivariate analysis elaborated that CR plus PR for induction immunochemotherapy and serum albumin $\geq 3.5 \mathrm{~g} / \mathrm{dL}$ were significantly associated with prolonged PFS in patients with non-cytotoxic maintenance (HR 0.31,95\% Cl 0.14-0.68, p = 0.004; HR $0.20,95 \% \mathrm{Cl} 0.064-0.64, \mathrm{p}=0.007)$. In contrast, in patients with cytotoxic maintenance, multivariate analysis was not performed because the $\mathrm{p}$-values for all variables were above 0.10 in univariate analysis.

Kaplan-Meier curves illustrated that responders of induction immunochemotherapy had significantly longer PFS of non-cytotoxic maintenance than non-responders (HR $0.31,95 \% \mathrm{Cl} 0.14-0.67$; log-rank $\mathrm{p}=$ 0.002) (Fig. 2A). Contrastingly, there was no significant difference in PFS associated with cytotoxic maintenance among responders and non-responders of induction therapy (HR 0.89, 95\% $\mathrm{Cl} 0.35-2.29$; log-rank $p=0.82$ ) (Fig. 2B). Similarly, the Kaplan-Meier curves for PFS in patients with cytotoxic and noncytotoxic maintenance were evaluated according to the responsiveness to prior immunochemotherapy. Among non-responders, the patients receiving cytotoxic maintenance had significantly longer PFS than did those with non-cytotoxic maintenance (HR 0.28, 95\% Cl 0.10-0.74; log-rank p $=0.007$ (Fig. $2 \mathrm{C}$ ). In contrast, among responders to prior immunochemotherapy, there was no significant difference in PFS between the two groups (HR 0.69, 95\% $\mathrm{Cl} 0.34-1.42$; log-rank $\mathrm{p}=0.31$ ) (Fig. 2D).

Furthermore, we analyzed the characteristics of prior immunochemotherapy responders and nonresponders who received non-cytotoxic maintenance. It was found that non-responders were more likely to have positive oncogenic driver mutations with NLR $\geq 5(20.0 \%$ vs $2.9 \%, p=0.075 ; 20.0 \%$ vs $2.9 \%, p=$ 0.075 , respectively) (Table 3). On assessing the association between responsiveness to prior 
immunochemotherapy and PFS duration with non-cytotoxic maintenance, it was found that patients with PFS > 90 days of non-cytotoxic maintenance had a significantly higher response rate to prior immunotherapy than did those with PFS $\leq 90$ days ( $89.2 \%$ vs $45.4 \%, p=0.001)$ (Fig. 3). These findings suggest that response to induction therapy might serve as a predictor for outcomes to non-cytotoxic maintenance therapy in NSCLC patients.

\section{Discussion}

Existing literature suggests that immunotherapy regimens, including those comprising ICls, contribute to long-term survival among advanced $\operatorname{NSCLC}$ patients $(17,18)$. Therefore, maintenance therapy following induction treatment plays the critical role of controlling the disease progression and enhancing patient survival. However, the impact of maintenance therapy among patients with advanced NSCLC has not been fully understood.

In this study, we identified a population of patients with advanced NSCLC who demonstrated good outcomes following administration of maintenance therapy after induction immunochemotherapy. Among non-responders to prior immunochemotherapy, it was noted that treatment with cytotoxic maintenance significantly prolonged PFS compared to those receiving non-cytotoxic maintenance. In contrast, there was no significant difference in PFS between responders receiving non-cytotoxic maintenance or cytotoxic maintenance. This is the first study to demonstrate the significance of response to prior immunochemotherapy as a potential predictor for the success of maintenance therapy after induction immunochemotherapy in patients with advanced NSCLC.

A previous meta-analysis demonstrated that response to ICls monotherapy was associated with good clinical outcomes, including long PFS for patients with advanced NSCLC. It also suggested that nonresponders to induction therapy might not be a suitable population for non-cytotoxic maintenance (19).

The present study found no difference in PFS for cytotoxic maintenance therapy between non-responders and responders to prior immunochemotherapy. Previous studies indicate that intervention by cytotoxic chemotherapy elicited antitumor immune systems through several mechanisms, including eliciting immunogenic tumor cell death, increasing the ratio between effector $T$ and regulatory $T$ cells (Tregs) in the peripheral blood, and reducing myeloid-derived suppressor cells in tumor tissues (20-22). Therefore, in the case of ineffectual induction immunochemotherapy, multidisciplinary maintenance, including chemotherapy, might be needed to enhance the effects of maintenance therapy. Although, the pemetrexed containing maintenance enhanced the efficacy of maintenance immunotherapy, there is a possibility of increased frequency of adverse effects, such as kidney injury, during long periods of maintenance therapy, leading to discontinuation of effective maintenance treatment $(12,23)$. Therefore, reduction of overmedication remains essential to drug tolerability along with long-term survival and economic viability. Future long-term follow-up studies are needed to support these findings.

Despite our best efforts, certain limitations of this study must be acknowledged. First, the distribution of histological subtypes was different between groups receiving cytotoxic and non-cytotoxic maintenance. 
Although there was no difference between histological subtypes and PFS for non-cytotoxic maintenance, the histological subtypes could potentially affect the comparison between maintenance therapies.

Second, we could not evaluate adverse effects associated with maintenance therapy which remains one of the most important factors to select therapeutic strategy in NSCLC patients. Third, a single experienced attending physician determined the timing of tumor imaging and judged the tumor response, which might introduce measurement bias.

In conclusion, the present study retrospectively analyzed patient data to identify potential predictors for maintenance therapy after induction immunochemotherapy in patients with advanced NSCLC. It found that response to induction immunochemotherapy was one of the most important factors to select a therapeutic strategy for maintenance therapy in patients with advanced NSCLC. Future prospective largecohort studies are warranted to confirm our findings.

\section{Declarations}

Funding: This research did not receive any specific grant from funding agencies in the public, commercial, or not-for-profit sectors.

Conflicts of interest: Tadaaki Yamada received commercial research grants from Pfizer, Ono Pharmaceutical, Janssen Pharmaceutical K.K., and Takeda Pharmaceutical Company Limited. Koichi Takayama received research grants from Chugai-Roche and Ono Pharmaceutical, and personal fees from AstraZeneca, Chugai-Roche, MSD-Merck, Eli Lilly, Boehringer-Ingelheim, and Daiichi-Sankyo. The other authors have no conflicts of interest to declare.

Availability of data and material: The datasets generated during the current study are not publicly available due to ethical constraints, but are available from the corresponding author on reasonable request.

Code availability: Not applicable.

Authors' contributions: Ryota Nakamura and Tadaaki Yamada contributed to the study conception and design. Ryota Nakamura, Kenji Morimoto, Akira Nakao, Yasuhiro Goto, Yuri Ogura, Takayuki Takeda, Chieko Takumi, Keisuke Onoi, Yusuke Chihara, Ryusuke Taniguchi, Takahiro Yamada, Osamu Hiranuma, and Satomi Tanaka obtained the clinical data. Data were interpreted by Ryota Nakamura, Tadaaki Yamada, Yoshie Morimoto, Masahiro Iwasaku, Shinsaku Tokuda, Yoshiko Kaneko, Junji Uchino, and Koichi Takayama. The manuscript was prepared by Ryota Nakamura and Tadaaki Yamada. The final version of the manuscript was read and approved by all the authors.

Ethics approval: The study protocol was approved by the Ethics Committee of Kyoto Prefectural University of Medicine and was conducted in accordance with the regulations on the handling of patients' personal information (Ethics Committee registration number: ERB309 C-1803). 
Consent to participate: Because this was a retrospective study, informed consent was waived and official website was used as an opt-out method, which was approved by the Ethics Committee of each individual hospital.

Consent for publication: Not applicable.

\section{References}

1. Bray F, Ferlay J, Soerjomataram I et al (2018) Global cancer statistics 2018: GLOBOCAN estimates of incidence and mortality worldwide for 36 cancers in 185 countries. CA Cancer J Clin 68:394-424. https://doi.org/10.3322/caac.21492

2. Ettinger DS, Aisner DL, Wood DE et al (2018) NCCN Guidelines Insights: Non-Small Cell Lung Cancer, Version 5.2018. J Natl Compr Canc Netw 16:807-821. https://doi.org/10.6004/jnccn.2018.0062

3. Planchard D, Popat S, Kerr K et al (2018) Metastatic non-small cell lung cancer: ESMO Clinical Practice Guidelines for diagnosis, treatment and follow-up. Ann Oncol 29:iv192-iv237. https://doi.org/10.1093/annonc/mdy275

4. Akamatsu H, Ninomiya K, Kenmotsu H et al (2019) The Japanese Lung Cancer Society Guideline for non-small cell lung cancer, stage IV. Int J Clin Oncol 24:731-770. https://doi.org/10.1007/s10147019-01431-z

5. Hellmann MD, Paz-Ares L, Bernabe Caro R et al (2019) Nivolumab plus Ipilimumab in Advanced Non-Small-Cell Lung Cancer. N Engl J Med 381:2020-2031. https://doi.org/10.1056/NEJMoa1910231

6. Paz-Ares L, Ciuleanu T-E, Cobo M et al (2021) First-line nivolumab plus ipilimumab combined with two cycles of chemotherapy in patients with non-small-cell lung cancer (CheckMate 9LA): an international, randomised, open-label, phase 3 trial. Lancet Oncol 22:198-211. https://doi.org/10.1016/S1470-2045(20)30641-0

7. Paz-Ares L, Luft A, Vicente D et al (2018) Pembrolizumab plus Chemotherapy for Squamous NonSmall-Cell Lung Cancer. N Engl J Med 379:2040-2051. https://doi.org/10.1056/NEJMoa1810865

8. Gandhi L, Rodríguez-Abreu D, Gadgeel S et al (2018) Pembrolizumab plus Chemotherapy in Metastatic Non-Small-Cell Lung Cancer. N Engl J Med 378:2078-2092. https://doi.org/10.1056/NEJMoa1801005

9. West $\mathrm{H}$, McCleod M, Hussein M et al (2019) Atezolizumab in combination with carboplatin plus nabpaclitaxel chemotherapy compared with chemotherapy alone as first-line treatment for metastatic non-squamous non-small-cell lung cancer (IMpower130): a multicentre, randomised, open-label, phase 3 trial. Lancet Oncol 20:924-937. https://doi.org/10.1016/S1470-2045(19)30167-6

10. Socinski MA, Jotte RM, Cappuzzo F et al (2018) Atezolizumab for First-Line Treatment of Metastatic Nonsquamous NSCLC. N Engl J Med 378:2288-2301. https://doi.org/10.1056/NEJMoa1716948

11. Paz-Ares LG, de Marinis F, Dediu M et al (2013) PARAMOUNT: Final overall survival results of the phase III study of maintenance pemetrexed versus placebo immediately after induction treatment 
with pemetrexed plus cisplatin for advanced nonsquamous non-small-cell lung cancer. J Clin Oncol 31:2895-2902. https://doi.org/10.1200/JC0.2012.47.1102

12. Visser S, Huisbrink J, van 't Veer NE et al (2018) Renal impairment during pemetrexed maintenance in patients with advanced nonsmall cell lung cancer: a cohort study. Eur Respir J 52.: https://doi.org/10.1183/13993003.00884-2018

13. de Rouw N, Boosman RJ, van de Bruinhorst $\mathrm{H}$ et al (2020) Cumulative pemetrexed dose increases the risk of nephrotoxicity. Lung Cancer 146:30-35. https://doi.org/10.1016/j.lungcan.2020.05.022

14. Paz-Ares L, de Marinis F, Dediu M et al (2012) Maintenance therapy with pemetrexed plus best supportive care versus placebo plus best supportive care after induction therapy with pemetrexed plus cisplatin for advanced non-squamous non-small-cell lung cancer (PARAMOUNT): a double-blind, phase 3, randomised controlled trial. Lancet Oncol 13:247-255. https://doi.org/10.1016/S14702045(12)70063-3

15. Diem S, Schmid S, Krapf M et al (2017) Neutrophil-to-Lymphocyte ratio (NLR) and Platelet-toLymphocyte ratio (PLR) as prognostic markers in patients with non-small cell lung cancer (NSCLC) treated with nivolumab. Lung Cancer 111:176-181. https://doi.org/10.1016/j.lungcan.2017.07.024

16. Kanda Y (2013) Investigation of the freely available easy-to-use software "EZR" for medical statistics. Bone Marrow Transplant 48:452-458. https://doi.org/10.1038/bmt.2012.244

17. Garon EB, Hellmann MD, Rizvi NA et al (2019) Five-Year Overall Survival for Patients With Advanced Non-Small-Cell Lung Cancer Treated With Pembrolizumab: Results From the Phase I KEYNOTE-001 Study. J Clin Orthod 37:2518-2527. https://doi.org/10.1200/JC0.19.00934

18. Gadgeel S, Rodríguez-Abreu D, Speranza G et al (2020) Updated Analysis From KEYNOTE-189: Pembrolizumab or Placebo Plus Pemetrexed and Platinum for Previously Untreated Metastatic Nonsquamous Non-Small-Cell Lung Cancer. J Clin Oncol 38:1505-1517. https://doi.org/10.1200/JC0.19.03136

19. Ye J, Ji X, Dennis PA et al (2020) Relationship Between Progression-Free Survival, Objective Response Rate, and Overall Survival in Clinical Trials of PD-1/PD-L1 Immune Checkpoint Blockade: A Meta-Analysis. Clin Pharmacol Ther 108:1274-1288. https://doi.org/10.1002/cpt.1956

20. Bracci L, Schiavoni G, Sistigu A, Belardelli F (2014) Immune-based mechanisms of cytotoxic chemotherapy: implications for the design of novel and rationale-based combined treatments against cancer. Cell Death Differ 21:15-25. https://doi.org/10.1038/cdd.2013.67

21. Wang Z, Till B, Gao Q (2017) Chemotherapeutic agent-mediated elimination of myeloid-derived suppressor cells. Oncoimmunology 6:e1331807. https://doi.org/10.1080/2162402X.2017.1331807

22. Roselli M, Cereda V, di Bari MG et al (2013) Effects of conventional therapeutic interventions on the number and function of regulatory T cells. Oncoimmunology 2:e27025.

https://doi.org/10.4161/onci.27025

23. Dumoulin DW, Visser S, Cornelissen R et al (2020) Renal Toxicity From Pemetrexed and Pembrolizumab in the Era of Combination Therapy in Patients With Metastatic Nonsquamous Cell NSCLC. J Thorac Oncol 15:1472-1483. https://doi.org/10.1016/j.jtho.2020.04.021 


\section{Tables}

Due to technical limitations, table 1,2,3 is only available as a download in the Supplemental Files section.

\section{Figures}

Fig 1

Combination of ICIs + chemo $(n=152)$

- Pemetrexed regimen $(n=66)$

- Non-pemetrexed regimen $(\mathrm{n}=86)$

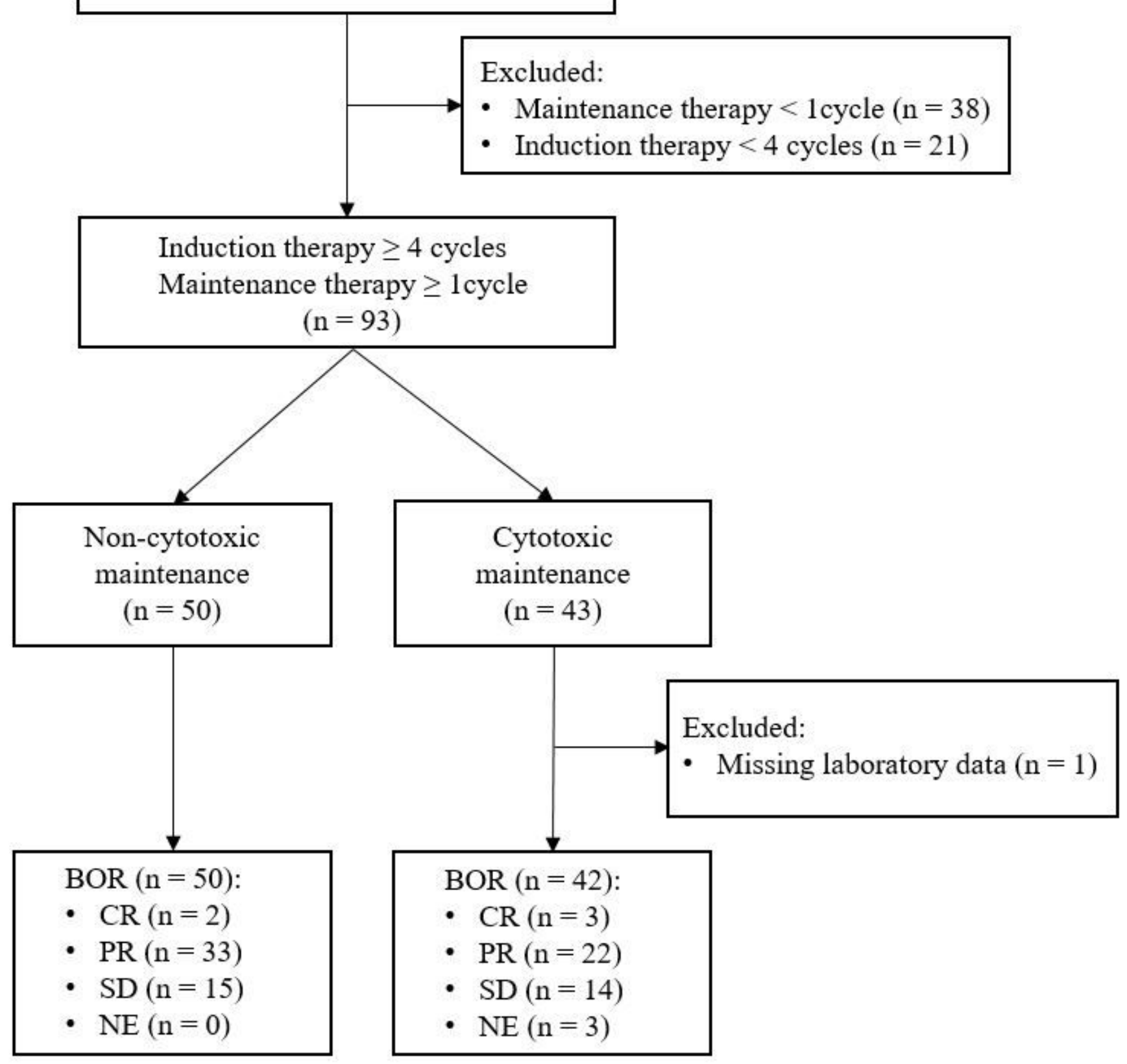

Figure 1 
Patient enrollment process and best overall response. BOR: best overall response, CR: complete response, PR: partial response, SD: stable disease, NE: not evaluable.

\section{Fig 2}

A

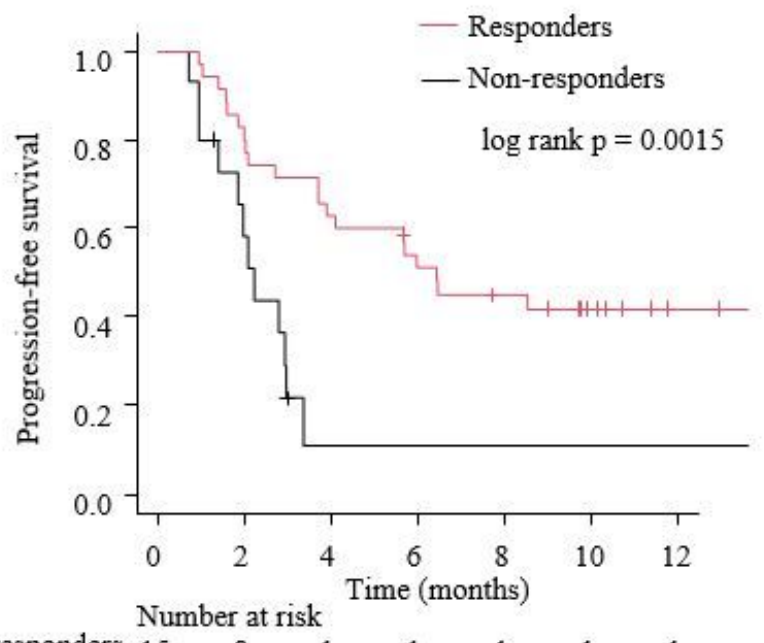

B

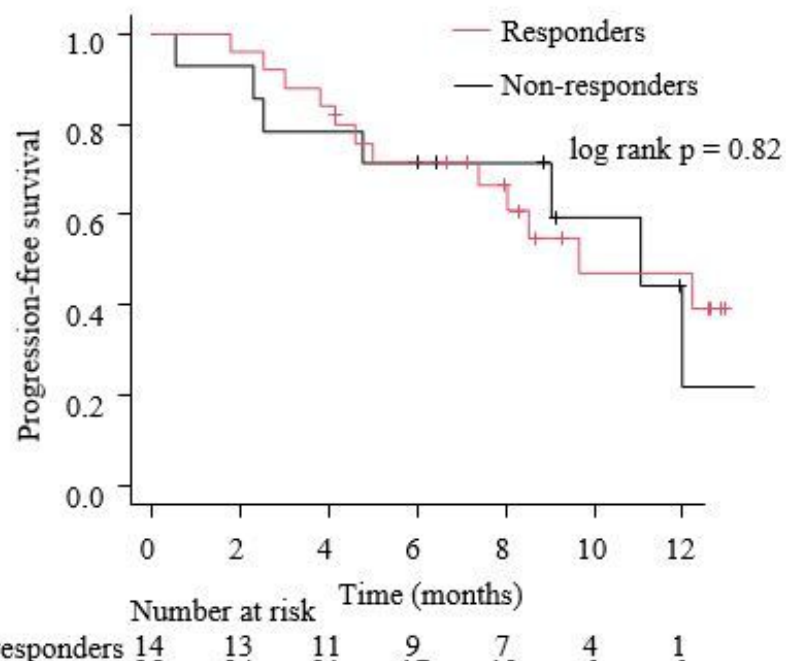

$\begin{array}{llllllll}\text { Non-responders } & 14 & 13 & 11 & 9 & 7 & 4 & 1 \\ \text { Responders } & 25 & 24 & 21 & 17 & 12 & 6 & 6\end{array}$

\section{$\mathrm{D}$}

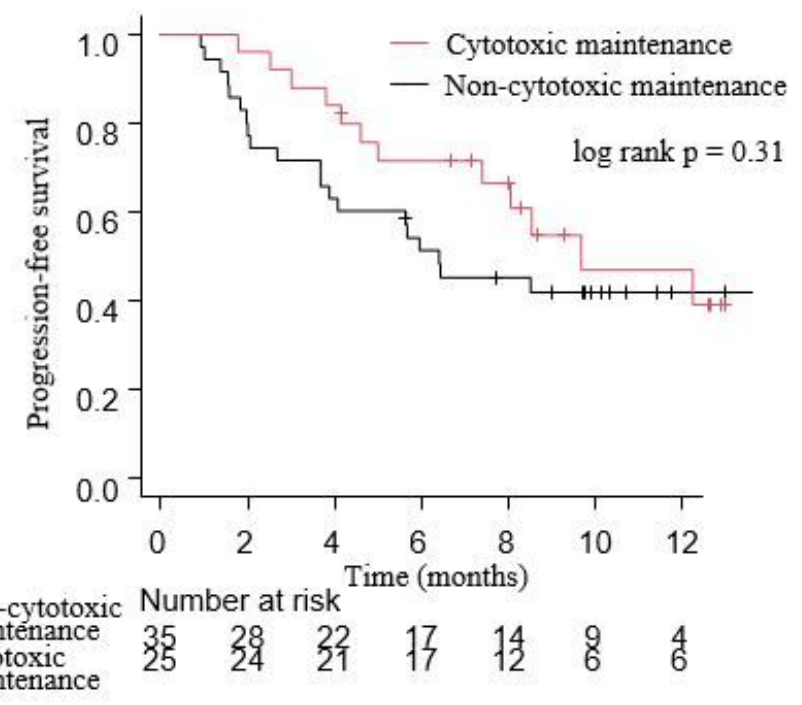

\section{Figure 2}

Kaplan-Meier curves for PFS of maintenance therapy in patients under non-cytotoxic maintenance (A) and those with cytotoxic maintenance (B) according to responsiveness to prior immunochemotherapy. 
Kaplan-Meier curves for PFS of maintenance therapy in non-responders (C) and responders (D) to prior immunochemotherapy according to maintenance regimens. PFS: progression-free survival.

\section{Fig 3}

PFS of maintenance therapy $>90$ days $\mathrm{SD}$

$$
\mathrm{n}=3
$$

$(10.7 \%)$

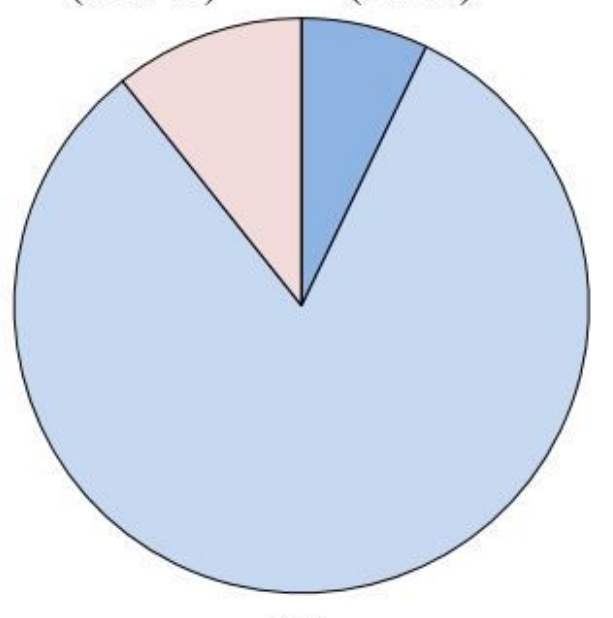

PR

$\mathrm{n}=23$

$(82.1 \%)$
PFS of maintenance therapy $\leq 90$ days

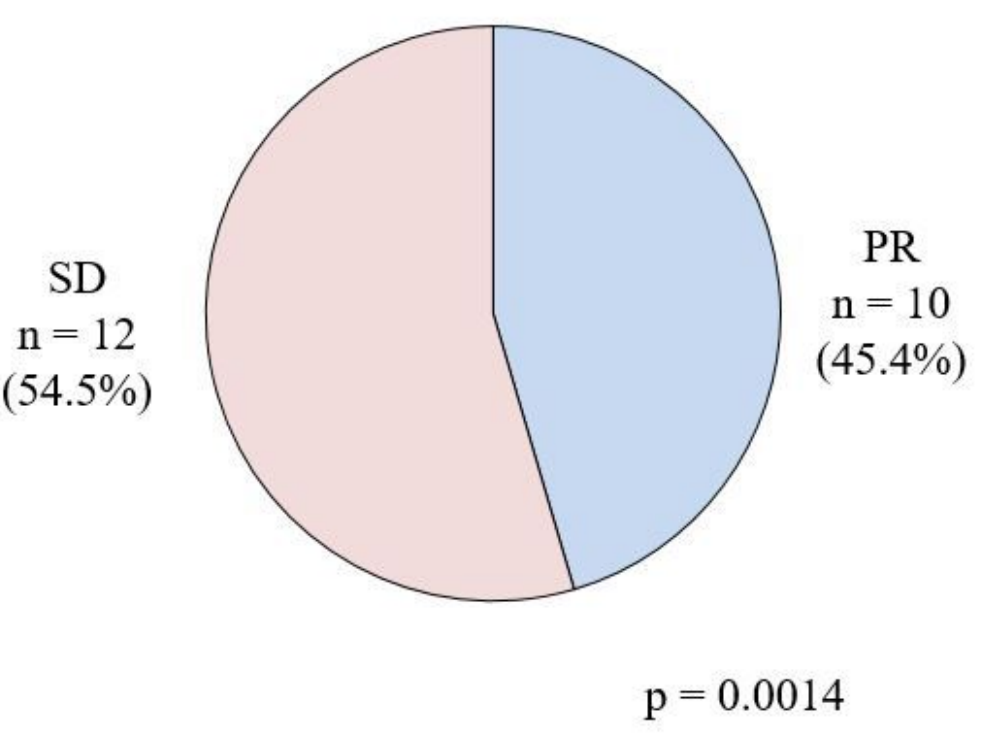

\section{Figure 3}

Best overall response to prior immunochemotherapy in patients with non-cytotoxic maintenance classified by PFS of maintenance therapy. CR: complete response, RP: partial response, SD: stable disease, PFS: progression-free survival.

\section{Supplementary Files}

This is a list of supplementary files associated with this preprint. Click to download.

- Table.pptx 